\title{
Patrimonio cultural europeo digitalizado: Europeana
}

\author{
European digital cultural heritage: Europeana
}

Patrimônio cultural digital europeu: Europeana

\author{
María-Antonia Garcia-Moreno' \\ Tony Hernández-Pérez"
}

Palabras clave:

\section{Resumen:}

\section{Europeana \\ Digitalización \\ Patrimonio cultural europeo \\ Biblioteca digital}

Se presentan las principales políticas, líneas de actuación, estrategias y marcos de referencia de la Unión Europea respecto a uno de los proyectos más importantes en materia de digitalización del patrimonio cultural europeo: Europeana. Se hace un recorrido por los derechos de autor y las 14 declaraciones de derechos que admite Europeana, y se pone en relación con el uso y reutilización de los objetos digitales. Se presentan los cuatro niveles de publicación que establece el Marco de Publicación de Europeana, así como la Estrategia de Contenidos que da prioridad a la calidad de lo digitalizado, más que a la cantidad, y a las necesidades de los usuarios. 


\begin{abstract}
:
The main policies, lines of action, strategies and reference frameworks of the European Union are presented with respect to one of the most important projects in the area of digitization of the European cultural heritage: Europeana. A tour of the copyright and the 14 rights declarations that Europeana admits is made, and it is related to the use and reuse of digital objects. The four levels of publication established by the Europeana Publication Framework are presented, as well as the Content Strategy that gives priority to quality of digitized material, rather than quantity and the need to improve usability and user needs.
\end{abstract}

Keywords:

Europeana

Digitization

European Cultural Heritage

Digital Library
Palavras-chave:

\author{
Europeana \\ Digitalização \\ Patrimônio cultural \\ europeu \\ Biblioteca digital
}

Resumo:

As principais políticas, linhas de acção, estratégias e estrutura de referência da União Europeia são apresentadas no que diz respeito a um dos projectos mais importantes no domínio da digitalização do património cultural europeu: Europeana. Uma revisão dos direitos autorais e 14 declarações de direitos que a Europeana admite serem feitas, e está relacionado ao uso e reutilização de objetos digitais. São apresentados os quatro níveis de publicação estabelecidos pelo Europeana Publication Framework, bem como a Estratégia de Conteúdo que prioriza a qualidade dos objetos digitalizados e não a quantidade e e usabilidade e as necessidades dos usuários. 


\section{Patrimonio cultural europeo digitalizado: Europeana}

\section{Introducción}

Unesco define el patrimonio cultural como "el legado de los artefactos físicos y los atributos intangibles de un grupo o sociedad heredados de generaciones pasadas, mantenidos en el presente y otorgado para el beneficio de las generaciones futuras" (UNESCO, 1972). Su visión del patrimonio se ha ido modificando con el tiempo y distingue entre:

- Patrimonio tangible: compuesto por patrimonio mobiliario como esculturas, pinturas, monedas o manuscritos; monumentos inmobiliarios, sitios arqueológicos y otros lugares y el patrimonio cultural subacuático.

- Patrimonio intangible: como las tradiciones orales, las artes escénicas, la artesanía o los rituales.

- Patrimonio natural: paisajes culturales y formaciones geológicas, biológicas o físicas.

- Patrimonio cultural en peligro de destrucción o por saqueo en conflictos armados.

Desde 2007, fecha en que se aprueba la Agenda Europea para la Cultura, el patrimonio cultural ha sido una prioridad para la Unión Europea. El reconocimiento a la importancia del patrimonio cultural se vio reflejado en las conclusiones del Consejo Europeo de mayo de 2014, al considerarlo como un recurso estratégico para una Europa sostenible. La Nueva Agenda europea para la Cultura comenzará en 2019 y no hace sino reforzar la idea de que es necesario "proteger y promover el patrimonio cultural europeo como recurso compartido, con el fin de sensibilizar a las personas en torno a nuestra historia y valores comunes y reforzar el sentido de la identidad común europea". (COMISIÓN EUROPEA, 2018b)

En Europa el patrimonio cultural pasó de entenderse como un conjunto de obras literarias, artísticas, lugares históricos, lenguas, costumbres y otros intangibles que son necesarios preservar y que suponen un coste, a reconocerse que el patrimonio cultural contribuye a una mayor cohesión social y produce beneficios económicos, no sólo en términos de turismo, sino que también contribuye al crecimiento económico, del empleo y de la sostenibilidad medioambiental (COMISIÓN EUROPEA, 2015). El patrimonio cultural se ha convertido pues en un elemento más de la Estrategia para el Mercado Único Digital en Europa.

Ese cambio de percepción, el patrimonio cultural como elemento que contribuye al desarrollo de una economía basada en lo digital, provocó el desarrollo de políticas sobre el patrimonio cultural digital de la Unión Europea (UE). Estas políticas se aplicaron en torno a tres ejes fundamentales:

1. La directiva del Parlamento Europeo y del Consejo relativa a la reutilización de la información del sector público cuya primera versión es de 2003, y que se basa en el principio general de que todos los documentos de bibliotecas, archivos y museos deberían ser reutilizables para propósitos comerciales y no comerciales, y que promueve que estos documentos estén disponibles en abierto, junto con sus metadatos, en formatos interoperables y con estándares abiertos PARLAMENTO EUROPEO; CONSEJO EUROPEO, 2018c) 
2. La recomendación de la Comisión sobre la digitalización y accesibilidad en línea del material cultural y la conservación digital, que insta a los estados miembros de la Unión Europea a tomar acciones para cooperar e involucrar al sector privado en la digitalización de su material cultural con el fin de incrementar la visibilidad del patrimonio cultural europeo y estimular el crecimiento de las industrias creativas en Europa (COMISIÓN EUROPEA, 2011).

3. Y toda la legislación relativa al copyright, incluyendo la normativa especial sobre obras huérfanas, que mencionaremos más tarde.

Alrededor de estas normas, la UE ha desarrollado un Plan de trabajo en materia de cultura (2015-2018) (CONSEJO EUROPEO, 2014), que subraya que la digitalización del contenido cultural y los servicios digitales pueden ayudar a promover la expansión de redes de turismo trans-europeo y una Nueva Agenda europea para la cultura, que también resalta el papel de la digitalización y la co-creación para superar las fronteras artísticas y económicas. (PARLAMENTO EUROPEO; CONSEJO EUROPEO, 2018c). Sobre estas bases, la UE ha financiado y puesto en marcha proyectos para asegurar el desarrollo de tecnologías y estándares para la digitalización, el acceso y el uso o la experiencia con el patrimonio cultural europeo. Y, al mismo tiempo, ha contribuido a financiar lo que posiblemente represente la infraestructura más importante en el campo de las bibliotecas: Europeana.

\section{Digitalización y derechos de autor}

Los antecedentes a los progresos de digitalización en Europa, con un análisis estadístico pormenorizado, incluyendo datos sobre costes de digitalización, los explican bien Agenjo y Campillejo (2015). La planificación y el seguimiento de las actividades de digitalización que se financiaban han sido aspectos claves en los avances de la digitalización en Europa y la encuesta ENUMERATE (JAN NAUTA; HEUVEL; TEUNISSE, 2017) ha permitido medir el progreso de la digitalización en los distintos estados miembros de la UE.

La recomendación sobre la reutilización de la información en el sector público (PARLAMENTO EUROPEO; CONSEJO EUROPEO, 2003) se convirtió en un instrumento útil para que los distintos gobiernos pusieran en marcha políticas y actividades coordinadas de digitalización. Los esquemas de planificación han sido diferentes: de acuerdo a un informe sobre el progreso de la implementación de la directiva (COMISIÓN EUROPEA, 2016), 10 estados miembros de la UE pusieron en marcha estrategias nacionales para incrementar el número de obras digitalizadas, otros 6 países optaron por estrategias ministeriales, más orientadas a temas específicos, mientras que algunos países impulsaron planes regionales o promocionaron estrategias de instituciones individuales con acuerdos con instituciones privadas (Google, Telefónica, FamilySearch, Kone Foundation, etc)

En cualquier caso, la estrategia de "libertad de organización" parece haber sido correcta, permitiendo que cada país se adaptara a su situación particular. Para algunos ha sido más sencillo optar por una política nacional común de digitalización, mientras que en otros se ha permitido a cada institución avanzar más lenta o rápidamente en función de su capacidad y de sus recursos. No parece haber grandes diferencias, al menos en cuanto a la cantidad comparada de digitalización, entre uno y otro enfoque. En todos los casos, lo que sí se pedía era el uso de estándares abier- 
tos y que todo lo digitalizado se pusiera accesible a través de Europeana, una especie de portal del patrimonio cultural europeo digitalizado.

Cada país de la UE ha tenido libertad para fijar sus prioridades de digitalización, basados en medidas, sobre todo, cuantitativas. En general, se puede decir que las cinco grandes prioridades, por orden, han sido:

1. Digitalización de recursos culturales de bibliotecas y archivos.

2. Digitalización de colecciones de museos.

3. Digitalización de monumentos, edificios históricos y lugares arqueológicos, algunos centrados en proyectos de digitalización 3D.

4. Digitalización del patrimonio audiovisual.

5. Digitalización de cultura intangible.

En enero de 2016 Europeana ya había alcanzado los 48,838,150 objetos digitalizados y había logrado, por tanto, su objetivo de 30 millones de ítems para final de 2015, que era lo que establecía la recomendación sobre digitalización (COMISIÓN EUROPEA, 2011). Los objetos audiovisuales digitalizados para esa fecha también habían alcanzado los $1,958,957$ objetos, el $98 \%$ del objetivo fijado para finales de 2015. A medida que se ha ido avanzando en la digitalización de contenidos ha sido necesario fijar unas mínimas características de calidad, que se han ido definiendo de acuerdo a las guías técnicas, los estándares y las especificaciones generadas en grandes proyectos, muchas de ellas accesibles hoy a través del proyecto Succeed (https://www.digitisation.eu/).

La digitalización de los fondos ha permitido el desarrollo de normas para la curación, la preservación y la restauración del patrimonio cultural. Ha permitido el acceso en línea a numerosas obras culturales, libros, cuadros, música y otros materiales, desde cualquier lugar y en cualquier momento. $Y$ ha permitido el desarrollo de material educativo, aplicaciones informáticas y otros proyectos para conocer y hacer uso de dicho patrimonio.

La estrategia para afrontar los proyectos de digitalización ha pasado por priorizar la digitalización de las obras que no ofrecían dudas sobre que ya estaban en el dominio público. El tiempo para que una obra, literaria, artística o científica, pase al dominio público varía de país a país, puede ir desde los 50 a los 100 años desde el fallecimiento del autor. En España, el tiempo para que una obra pase al dominio público es de 70 años a partir de la muerte del autor. Y, por tanto, una de las primeras fases de cualquier proyecto debería consistir en identificar qué obras están ya en dominio público.

En los proyectos de digitalización, la existencia de obras que las instituciones culturales consideraban que deberían digitalizarse hicieron aflorar muy pronto la existencia de numerosas obras huérfanas, un patrimonio cultural riquísimo sobre el que resultaba muy difícil averiguar a quién correspondía los derechos de autor. Para solventar la situación la UE aprobó una directivasobre ciertos usos autorizados de las obras huérfanas (CONSEJO EUROPEO, 2012) para propiciar su digitalización y puesta a disposición en línea con todas las garantías legales para los archivos y bibliotecas.

El problema de estas obras huérfanas, protegidas por derechos de autor, pero cuyos autores $u$ otros titulares de derechos no pueden ser identificados o localizados, ha sido de tal importancia que la EUIPO, la Oficina de Propiedad Intelectual de la Unión Europea, ha crea- 
do una base de datos de obras huérfanas, la Orphan Works Database. Esta base de datos facilita "información sobre las obras huérfanas contenidas en las colecciones de bibliotecas, centros de enseñanza y museos accesibles al público, así como archivos, organismos de conservación del patrimonio cinematográfico o sonoro y organismos públicos de radiodifusión establecidos en los Estados miembros" (EUIPO, 2018).

La base de datos permite, tanto hacer uso de obras huérfanas en proyectos de digitalización, como identificar y dar de alta aquellas obras huérfanas descubiertas. $\mathrm{Y}$, al mismo tiempo, sirve para que los titulares de derechos de alguna obra huérfana puedan ponerse en contacto con las organizaciones que están haciendo uso de ellas y poner fin a su condición de obras huérfanas reclamando sus derechos. Existen otras bases de datos que también contribuyen a resolver dudas sobre las obras huérfanas, como ARROW (AccessibleRegistriesofRightsInformation), IDA (International Documentationon Audiovisual works) y otras.

\section{Acceso y reutilización de los conteni- dos digitalizados}

La digitalización cobra todo su sentido cuando no sólo se preserva sino cuando los contenidos digitalizados se ponen a disposición del público para su utilización y reutilización, sin restricciones. Poner accesible los contenidos digitalizados ha supuesto nuevos retos para los archivos y las bibliotecas, como superar el miedo a pensar que la digitalización hace que se pierdan usuarios que visitan físicamente la biblioteca o el temor a que otros puedan copiar la obra digitalizada. Afortunadamente, ya casi nadie sigue usando las invasivas marcas de agua. Pero, probablemente, el reto principal ha sido el de resolver la incertidumbre legal sobre los posibles derechos de autor cuando los trabajos eran digitalizados.

Las estrategias para promocionar el acceso y la reutilización de los contenidos digitalizados por las instituciones europeas pasan por:

- Asegurar, mediante las licencias adecuadas, que el material que ya está en dominio público permanecerá en el dominio público.

- Animar a todas las instituciones a compartir, al menos los metadatos de estos objetos culturales, mediante licencias CCO.

- Promover el acceso más amplio posible de los materiales digitalizados y en dominio público para su reutilización, con o sin propósitos comerciales.

- Implicar a los usuarios a través de visitas virtuales, exposiciones virtuales, visualizaciones 3D para colaborar con instituciones educativas o centros de innovación o capacitación de tal forma que se utilicen los materiales digitalizados, incentivándolo, a veces, con premios, o con concursos que sirvan para estimular la innovación en el uso de estos materiales.

- Publicitar su existencia y su uso a través de wikis, blogs, hackathones, páginas web o campañas en prensa o en medios sociales, como lo ha hecho la Biblioteca Nacional de España con la serie "El Ministerio del Tiempo" (RODRÍGUEZMATEOS; HERNÁNDEZ-PÉREZ, 2015).

- Tomar las medidas que sean necesarias para limitar el uso de marcas de agua u otras protecciones visuales que reduzcan la usabilidad del material digitalizado.

- Estimular a todas las instituciones culturales, editores y propietarios de derechos para que utilicen Europeana como plata- 
forma para poner ese material digitalizado disponible para todo el mundo.

La necesidad de ser interoperable con Europeana y con el resto de instituciones culturales ha estimulado el crecimiento de acuerdos, a escala nacional o regional, para tener recomendaciones y directrices comunes sobre los procesos de digitalización, los formatos o los metadatos. Europeana ha promovido también el acceso a sus contenidos a través de APIs y de forma experimental en formato de datos abiertos enlazados, lo que ha permitido nuevas y creativas formas de acceder al contenido a través de apps y otros programas, muchos de ellos específicamente orientados hacia las escuelas para reutilizar todos estos recursos.

La reducción de la usabilidad de algunos materiales digitalizados ha sido, hasta ahora, norma común. Muchas veces, las imágenes se ofrecen con marcas de agua o a baja resolución y si alguien desea imágenes a una resolución mayor o sin marcas, se cobra por el servicio. No se trata tanto de un tema de derechos sino de cobrar por un servicio, lo que es especialmente conveniente para los ingresos de algunos museos.

Afortunadamente, aunque aún demasiado despacio, esta práctica va decayendo. Por ejemplo, el Rijksmuseum de Amsterdam ya permite a todo el mundo la reutilización gratuita de su material digitalizado de dominio público en formato de alta resolución. No ha sido la única institución, también el Museum für Kunstund Gewerbe (MKG) de Hamburgo ha hecho algo parecido, aunque estos ejemplos siguen siendo excepcionales.

\section{Preservación del material digitalizado}

Las estrategias de preservación a largo plazo del material digitalizado en la
UE difieren de país a país. Alemania y Suecia han creado organismos específicamente dedicados a este fin mientras que en la mayoría de países se limitan a poner en marcha esquemas o planes de preservación limitados a algún sector o institución específica. En España, por ejemplo, el Ministerio de Cultura dispone de una Política de gestión de documentos electrónicos "con directrices, esquema de metadatos, estándares, etc. para la gestión de los documentos y expedientes electrónicos (...) con el fin de garantizar su preservación para generaciones futuras y facilitar el acceso a la información pública", (MINISTERIO DE CULTURA, 2016), pero no se aplica en otros ministerios.

La política de preservación digital del Archivo Nacional en España está basada en el mantenimiento de múltiples copias en formato digital (cintas LTO3) y en formato analógico (microfilm de imágenes digitales) así como migraciones y conversiones periódicas de formato. Pero, como decíamos anteriormente, lo que puede funcionar para un ministerio no se aplica en otro y lo que se hace en un país de la UE aún difiere, en cuanto a preservación, de lo que se hace en otros.

La mayor parte de los países han comenzado a dictar normas para permitir cambios de formato y migraciones digitales de material cultural con fines específicos de preservación, así como una amplia preocupación por los proyectos de preservación de los contenidos web a largo plazo, como el Archivo de la Web Española (http://www.bne.es/ es/Colecciones/ArchivoWeb/). Sin embargo, excepto para el material fílmico, aún es necesario reforzar la legislación sobre el depósito legal de materiales nacidos en formato digital con el fin de garantizar su preservación a largo plazo. En España, por ejemplo, está ya regulado por el Real Decreto 635/2015, 
de 10 de julio, sobre el depósito legal de las publicaciones en línea, pero aún faltan algunos países por tener una legislación similar.

Merece la pena destacar la existencia de centros de competencia digital en materia de digitalización, nacidos al amparo de diferentes proyectos europeos. Tres grandes ejemplos de estas iniciativas en donde se intercambia información muy valiosa son: PrestoCentre (https://www.prestocentre.org/) para el material audiovisual; el ya mencionado Impact, para el material textual (https:// www.digitisation.eu/); o el DCH-RP Digital Cultural HeritageRoadmapforPreservation (http://www.dchrp.eu/)

\section{Europeana}

Justo ahora, en 2018, se cumplen diez años de la puesta en funcionamiento de Europeana, proyecto europeo común que da acceso online a su patrimonio digital (CONSEJO EUROPEO, 2008). La política europea de digitalización del patrimonio promueve que una vez digitalizado los objetos digitales estén, además, accesibles a través de Europeana, una plataforma que permite el acceso a ese patrimonio digital. Europeana proporciona acceso a más de 58 millones de objetos digitales (texto, imagen, sonido, vídeo y material en 3D) procedentes de 3700 bibliotecas, archivos, museos, galerías y colecciones audiovisuales, que conforman el patrimonio cultural europeo depositado en instituciones de memoria y cultura de los países miembros de la Unión.

En su forma actual, Europeana es una plataforma de internet que permite el acceso multilingüe al patrimonio cultural digital perteneciente a instituciones del patrimonio cultural... cuya finalidad es crear valor de cara a los usuarios finales, los Estados Miembros, las instituciones del patrimonio cultural, la reutilización creativa y con fines de investigación. (CONSEJO EUROPEO, 2016)

Las políticas europeas relativas al patrimonio cultural digital y digitalizado se han apoyado en acciones, directrices, recomendaciones y resoluciones promovidas al amparo de las instituciones de la Unión: Consejo (CONSEJO EUROPEO, 2014), Comisión (COMISIÓN EUROPEA, 2011) y Parlamento Europeo (PARLAMENTO EUROPEO, 2010), y tienen como base los Artículos 167.1 y 167.4 del Tratado de Funcionamiento de la Unión Europea, donde se manifiesta el compromiso de contribuir al florecimiento de las culturas de los Estados Miembros, poniendo de relieve el patrimonio cultural común, respetando y fomentando la diversidad de las culturas.

En 2016, el Consejo de la Unión Europea hacía público los avances conseguidos en la digitalización, acceso online y conservación digital del patrimonio cultural (CONSEJO EUROPEO, 2016), estimando, según datos de ENUMERATE (ENUMERATE, 2014), que se había digitalizado el $10 \%$ del patrimonio cultural europeo y un tercio del mismo disponible online. Gracias al trabajo de las instituciones del patrimonio cultural y de los grupos de expertos que han intervenido en su desarrollo, Europeana ha sido capaz de promover la aceptación, desarrollo y uso de normas y modelos para compartir contenidos y metadatos.

Desde el proyecto inicial de Europeana hasta ahora, se han ido sumando a las bibliotecas, archivos y museos, otras instituciones como proveedoras de materiales digitales: filmotecas, fonotecas, productoras de radio y televisión, galerías de arte, incluso algunas aportaciones de ciudadanos europeos: fo- 
tos, cartas y memorias orales (AGENJO BULLÓN; HERNÁNDEZ CARRASCAL, 2018). Las prioridades de actuación de Europeana, además de las dirigidas a las instituciones de memoria y cultura, se han extendido a otros sectores: educativos, de investigación, industria creativa o turismo. De hecho, se presenta como una plataforma cuyos contenidos pueden ser utilizados por un abanico amplio de usuarios, no solo profesionales de las instituciones de memoria y cultura, sino por cualquier ciudadano interesado en alguna de sus colecciones.

Europeana tiene como objetivo primordial facilitar el conocimiento a la cultura y la historia de Europa y a ello debe añadir el de conectar todo el patrimonio cultural europeo, lo que supone, por ejemplo, que las instituciones culturales puedan compartir colecciones $y$, al mismo tiempo, que se puedan reutilizar e intercambiar sus contenidos, siempre respetando los derechos de autor. De manera que en el camino recorrido contabiliza muchos avances, algunos se han convertido en valores de marca, como el modelo europeo de datos EDM, exportado a proyectos no europeos como la Digital Public Library ofAmerica (DPLA); pero también debe afrontar importantes retos relacionados con la calidad de los contenidos, la posibilidad de compartir y reutilizar los datos, la facilidad de uso, la visibilidad, declaraciones de dominio público y derechos de autor, su propia gestión, mantenimiento y financiación.

\section{EI Marco de Publicación de Europeana (MPE)}

Para apoyar la visión de "transformar el mundo a través de la cultura" (EUROPEANA, 2015), Europeana trabaja para que los contenidos que ofrece sean atractivos, de calidad, útiles, de fácil descubrimiento, uso y acceso rápido. Por ello pide a los proveedores de datos que proporcionen buenos metadatos y, siempre que sea posible, el acceso a los objetos digitales. Es por esto por lo que la información, para que pueda ser incluida en Europeana debe presentarse siguiendo el modelo de datos EDM(EUROPEANA, 2017b), un formato de agregación que se emplea en Europeana Collections y cuyas especificaciones aparecen en la Guía de publicación de Europeana (EUROPEANA, 2017a). De tal forma que información relevante como puede ser el acceso al objeto digital se facilite a través de elementos del modelo EDM tales como:

edm: object El enlace al objeto se utiliza para crear una vista previa

edm: is Shown At Enlace a un sitio web que contiene el objeto digital

edm: is Show By Enlace directo al objeto digital

edm: rigths La declaración de derechos que se haya definido para el objeto

Estos elementos que identifican el acceso al objeto digital se relacionan con los cuatro niveles o escenarios de publicación que establece el Marco de Publicación de Europeana (MPE) con el slogan "lo que das es lo que obtienes" (EUROPEANA, 2016):

Nivel 1. Europeana como motor de búsqueda. "Quiero que la gente encuentre mis colecciones". Este escenario está pensado para aquellas instituciones que quieren estar más visibles y localizables a través de la plataforma de europeana. eu pero de momento prefieren que sus contenidos no estén incluidos en Europeana Collections. Utilizan Europeana para atraer a más usuarios y conseguir más tráfico hacia sus sitios web. En este 
escenario las instituciones deben facilitar una vista previa al objeto digital de al menos 400 píxeles de ancho. Lo que obtienen las instituciones en este Nivel 1 de participación son más referencias y tráfico hacia sus sitios web.

Nivel 2. Europeana como escaparate. "Quiero que la gente encuentre y visualice mis colecciones en Europeana". En este nivel de publicación los contenidos forman parte de las colecciones temáticas que ofrece Europeana. El usuario no tiene que ir a otro sitio web para visualizar, consultar o descargar el objeto digital, sino que lo hace en el contexto de una colección de Europeana (Moda, Arte, Deportes, Música...). La institución proporciona un enlace directo a la imagen (objeto digital) con un ancho mínimo de 800 píxeles. Lo que obtienen las instituciones en este Nivel 2 de participación es una mayor presencia en Europeana Collections.

Nivel 3. Europeana como plataforma de distribución. "Quiero que la gente encuentre, visualice y utilicen mis colecciones, pero sin beneficios comerciales". Las colecciones se visualizan en Europeana Collections con un nivel de calidad alta para que puedan reutilizarse, se recomienda 1200 píxeles de ancho para imágenes. Los proveedores ofrecen objetos digitales con licencias que permiten la reutilización no comercial. Pueden incorporarse a proyectos y colaboraciones en los que está inmersa Europeana (Historiana, Europeana Research...). Lo que obtienen es una mayor presencia en Europeana y en otros sitios web y servicios no comerciales.

Nivel 4. Europeana como plataforma de libre reutilización. "Quiero que la gente encuentre, visualice y utilice mis colecciones como quieran". Las colecciones deben estar libres de derechos, por lo que deben disponer de la corres- pondiente declaración de derechos que permita su libre reutilización. Los datos pueden ser utilizados en plataformas abiertas como Wikimedia y en redes sociales; pudiendo incorporarse a otros proyectos (Europeana Labs, Field Trip...). Lo que obtienen es una gran difusión de sus contenidos, tanto en el contexto de Europeana Collections como fuera de ella, en servicios comerciales y no comerciales.

En todos los niveles de publicación, las instituciones proveedoras de contenidos se hacen más visibles y se localizan fácilmente en la medida que su información está indexada en los principales motores de búsqueda y disponible en un formato estándar. Además, se benefician de la tecnología Linked Data que emplea Europeana, de manera que cuando alguien busque en Europeana contenido relacionado con alguna institución proveedora de información, aparecerá relacionado con ella.

Con el Marco de Publicación Europeana (MPE) pretende animar a las instituciones a que compartan sus colecciones atendiendo a las estrategias digitales de cada institución, a la calidad de los contenidos y a sus derechos de autor. A través de la Guía de Publicación de Europeana, las instituciones pueden saber qué requisitos de calidad deben cumplir para cada nivel o escenario. Aquellas instituciones que deseen compartir sus colecciones en todos los escenarios deben cumplir requisitos de alta calidad y reutilización de sus datos.

\section{Declaraciones de derechos y Marco de licencias de Europeana}

Para determinar si un objeto digital pertenece al Nivel 3 o 4 de publicación y reutilización, el Marco de Publicación de Europeana (MPE) se basa en una lista de 14 declaraciones de derechos recogidas 
en el Marco de Licencias de Europeana (EUROPEANA, 2011), de las cuales, nueve permiten a los usuarios la reutilización de los objetos digitales. Cuatro de ellas sin restricciones ni condiciones:

\section{Marca de Dominio Público (PDM).}

2. CC0 1.0 Ofrecimiento al Dominio Público Universal (CCO).

3. Licencia Creative Commons de Reconocimiento (CC BY). En la que se debe hacer referencia al autor original.

4. Licencia Creative Commons de Reconocimiento-Compartir Igual (CC BY-SA). Con la obligación de hacer referencia al autor original. En el caso de que se distribuyan trabajos derivados se debe hacer bajo la misma licencia.

Hay otras cinco declaraciones que permiten la reutilización pero que pueden tener restricciones:

1. Licencia Creative Commons de Reconocimiento No Comercial (CC BY-NC). Permite la reutilización no comercial del objeto siempre que se haga referencia al autor original.

2. Licencia Creative Commons de Reconocimiento-Compartir Igual (CC BYNC-SA). Permite la reutilización no comercial del objeto siempre que se haga referencia al autor original. Si se distribuyen trabajos derivados se debe hacer bajo la misma licencia.

3. Licencia Creative Commons de Reconocimiento-No Comercial-No Derivadas (CC BY-NC-ND) permite el intercambio no comercial del objeto digital siempre que se haga referencia al autor original. No se permiten modificaciones de la obra.

4. Licencia CreativeCommons de Reconocimiento-No Derivadas (CC BY-ND) permite compartir el objeto haciendo referencia al autor original. No se permiten modificaciones del objeto.

5. Licencia de Reutilización No Comercial, fuera del derecho de autor (OOC$\mathrm{NC})$ permite la reutilización no comercial del objeto.

A través de este Marco de Licencias, Europeana normaliza y armoniza la información y las prácticas relacionadas con la propiedad intelectual. Asimismo, proporciona a los usuarios la información necesaria para saber lo que pueden y no pueden hacer, tanto con los metadatos como con los contenidos que aparecen en la plataforma europea, basándose en dos principios:

- Que todos los metadatos agregados para ser publicados en Europeana puedan ser utilizados libremente sin restricción alguna. Para cumplir este principio Europeana pone todos los metadatos bajo los términos de Dedicación de Dominio Público CCO.

- Respecto a los contenidos, que atendiendo a la lista de declaraciones de derechos se describa el estado de los derechos de los objetos digitales que los proveedores de información ofrecen a través de Europeana.

Los cuatro elementos que forman el Marco de Licencias son los que aseguran que los metadatos y los contenidos pueden ser agregados y libremente reutilizados por terceros:

1. Acuerdo de Intercambio de Datos de Europeana (DEA)

2. Creative Commons 0 Dominio Público Universal (CCOwaiver) / Europeana Guía de Uso de Datos

3. Términos para las contribuciones de usuarios 
4. edm: rights campo de Europeana Data Model

Estos cuatro elementos constituyen la base de trabajo en la que las instituciones proveedoras de objetos digitales se apoyan para cumplir con el requisito de informar con claridad a los usuarios acerca de todas las cuestiones relacionadas con los derechos de propiedad intelectual de todos aquellos objetos digitales que depositan en la plataforma europea.

\section{Estrategia de Contenidos de Europeana: la calidad y los usuarios}

Basándose en las tres prioridades de la Estrategia de Europeana: datos de calidad, datos abiertos y datos que aporten valor; y siguiendo el Marco de Publicación de Europeana (MPE) con los cuatro escenarios para publicar y compartir información, la Estrategia de Contenidos de Europeana (EUROPEANA, 2018)establece como principio número uno la calidad por encima de la cantidad, lo que significa que:

- Se debe utilizar el MPE para crear contenidos de calidad alta, así como instar a las instituciones para que abran sus datos.

- Se prioriza la mejora de los datos ya incluidos más que añadir datos nuevos.

- Se deben eliminar los datos que no cumplan con lo especificado en la Guía de Publicación de Europeana.

- Se promueva la exhibición de los datos de gran calidad en las Colecciones Temáticas.

- Se consiga que la mejora de la calidad de los datos sea la prioridad número uno de la Estrategia de Europeana
- Que en 2020 el número de contenidos del Nivel 2 y superiores sea mayor que el del Nivel 1.

El otro principio de la Estrategia de Contenidos da prioridad a lo que los usuarios piden y necesitanen el momento de tomar decisiones respecto a la selección y publicación de contenidos. Para cumplir con este principio, pone de manifiesto la necesidad de analizar las estadísticas de uso de los contenidos, la realización de encuestas y entrevistas a los usuarios para identificar sus necesidades de información, así como la evaluación de los nuevos contenidos en relación con el cumplimiento del MPE, y la adecuación a las Colecciones Temáticas. En este sentido, Europeana pretende identificar a otros usuarios diferentes de las instituciones de memoria y cultura tradicionales; a usuarios potenciales; y para ello busca otros canales o fuentes que puedan facilitarle este tipo de información, y que van más allá de los resultados que obtiene en las encuestas, ya sean usuarios estratégicos por razones económicas, de marca, o por tendencias.

Los proveedores saben, a través de la Guía de Publicación de Europeana, que la calidad de los datos es lo que determina que una colección digital se publique. Si los metadatos no cumplen con los requisitos establecidos en la Guía, los datos no se publicarán. Pero no es la única razón, pues existen no pocos objetos digitales de los siglos XX y XXI que no pueden estar accesibles, ni en esta, ni en otras plataformas, por estar sujetos a derechos de autor. Un problema en el que Europeana se emplea a fondo para resolver y poder así favorecer el uso y la reutilización de contenidos en sectores como el de la educación. Se puede decir que, al menos en el Nivel 1 de publicación, Europeana ha conseguido que contenidos con restricciones 
puedan ser accesibles a través de Europeana Collections.

Según datos de la propia Europeana, a finales de 2016, aproximadamente el $83 \%$ de los contenidos se ajustaban al Nivel 1 y solo el $17 \%$ al Nivel 2 o superiores de publicación. La intención para el 2020 es la de invertir el predominio de los niveles de publicación de contenidos para conseguir un $80 \%$ en el Nivel 2 y superiores y reducir a un $20 \%$ el Nivel 1 de publicación (EUROPEANA, 2018; p.21).

A partir de los principios de calidad y demanda de los usuarios, la Estrategia de Contenidos determina, junto con los proveedores, cuestiones tales como si vale la pena un determinado contenido, así como la integración en Europeana de metadatos procedentes de proyectos colaborativos como Wikimedia Foundation, e incluso la admisión de contenido colaborativo para conseguir una mayor participación de los usuarios.

En lo que a cantidad se refiere, en octubre de 2017, España contaba con 629 proyectos de digitalización procedentes de la iniciativa de instituciones culturales, 215 repositorios OAI y 7,3 millones de objetos digitales. Se sitúa a la cabeza como proveedor de datos a través de Hispana, además de aparecer como uno de los principales usuarios junto a los Estados Unidos de América, Holanda, países nórdicos, Alemania, PoIonia, Reino Unido (GONZÁLEZ, 2018). Sin embargo, frente al volumen de registros y objetos digitales, tanto de Hispana, proveedor español, como la propia Europeana, no son proyectos suficientemente conocidos, ni en el ámbito educativo, ni como repositorio útil para investigación, y mucho menos entre el público en general. En cambio, sí lo son para los profesionales de bibliotecas, archivos y museos, que reconocen y valoran el gran avance conseguido a través del proyecto común europeo de digitalización de la memoria y la cultura, a pesar de resaltar de forma recurrente la mala calidad de la interfaz de búsqueda de la plataforma Europeana.

Ya en 2014 el estudio realizado por User Intelligent puso de manifiesto que tanto los datos que se ofrecían como la manera en la que se servían no satisfacían a los usuarios encuestados(MAK; BRINKMAN, 2014). Recientemente, Europeana ha realizado una consulta pública sobre el uso de la plataforma (COMISIÓN EUROPEA, 2018a)con el fin de identificar necesidades de información de los usuarios, objetos y colecciones con mayor trafico y éxito de consulta, pero también otros canales y otras fuentes diferentes a las instituciones culturales tradicionales.

\section{Conclusiones}

La Unión Europea ha hecho grandes inversiones para la digitalización de su patrimonio cultural. Desde 2007, en Europa se concibe el patrimonio cultural como un elemento vertebrador que contribuye a la cohesión social de Europa y, además, como un activo que produce beneficios económicos, en el turismo y en la creación de industrias culturales, por lo que se ha convertido en un elemento más de la Estrategia para el Mercado Único Digital en Europa.

La política sobre el patrimonio cultural europeo se enmarca dentro de la estrategia global para este Mercado Único Digital en Europa y ha girado en torno a tres grandes normas:

a. La directiva relativa a la reutilización de la información del sector público, que obliga a que todos los documentos generados en la administración pública, no sujetos a secretos de estado o que afecten a la privacidad de las personas, deberían ser 
reutilizables, incluidos los de las bibliotecas, archivos y museos, para propósitos comerciales y no comerciales.

b. La recomendación sobre la digitalización y accesibilidad en línea del material cultural y la conservación digital, que insta a los estados miembros de la Unión Europea a tomar acciones para cooperar e involucrar al sector privado en la digitalización de su material cultural con el fin de incrementar la visibilidad del patrimonio cultural europeo y estimular el crecimiento de las industrias creativas en Europa.

c. Y toda la legislación relativa al copyright, lo que permite a las instituciones, por un lado, poner en acceso abierto aquellas obras libres de derechos $y$, por otra, ofreciendo a las instituciones las garantías jurídicas necesarias para que los proyectos de digitalización no se interrumpieran, incluso cuando había obras huérfanas sobre las que existen dudas sobre la propiedad de los derechos. No sólo se legisló sobre estas obras huérfanas sino que la Oficina de Propiedad Intelectual de la Unión Europea, ha creado una base de datos de obras huérfanas, la Orphan Works Database que permite identificar y dar de alta aquellas obras huérfanas descubiertas y, al mismo tiempo, para que los titulares de derechos de alguna obra huérfana puedan ponerse en contacto con las organizaciones que están haciendo uso de ellas y poner fin a su condición de obras huérfanas reclamando sus derechos.

La Unión Europea ha dejado total libertad para que cada país definiese sus prioridades en cuanto a qué fondos digitalizar y cada país estableció su propia política de planificación de la digitalización, a nivel estatal, autonómico o incluso una política independiente por cada institución. En todos los casos, lo que sí se exigía era el uso de estándares abiertos y que todo lo digitalizado se pusiera accesible a través de Europeana.

En general, se puede afirmar que los primeros recursos culturales digitalizados fueron los de las colecciones de bibliotecas, archivos y museos. Los primeros objetivos definidos estuvieron basados en medidas cuantitativas, digitalizar, cuanto más, mejor. Los objetivos cuantitativos se cumplieron a principio de 2016 y desde entonces se ha optado más por una política de calidad de los metadatos y de los objetos digitalizados que por seguir aumentando la cantidad del patrimonio digitalizado.

El proceso de digitalización ha traído consigo el desarrollo de estándares y estrategias de preservación, de interoperabilidad y de contenido para compartir y promocionar el acceso y la reutilización de los contenidos digitalizados por las instituciones europeas. Europeana, la plataforma digital europea en donde se vuelca la mayor parte del patrimonio digitalizado, marca, además, las directrices sobre estos estándares, como el modelo europeo de datos EDM (Europeana Data Model) o el Marco de Publicación de Europeana, un documento en el que se establecen cuatro escenarios (niveles) para compartir colecciones con Europeana basados en la oferta y las necesidades de los proveedores de datos y en una lista de 14 declaraciones de derechos recogidas en el Marco de Licencias de Europeana.

Aunque se ha hecho mucho, aún queda mucho por hacer. Por ejemplo, muchos usuarios en Europa desconocen la existencia de Europeana, aquellos que sí la conocen manifiestan que la usabilidad del sitio aún tiene un gran recorrido de mejora y el fijar objetivos cuantitativos como medidas de evaluación ha provocado que persistan metadatos y objetos digitalizados con una calidad menor a la deseada. 


\section{Referencias}

AGENJOBULLÓN, X.; HERNÁNDEZCARRASCAL, F. Diez años de Europeana. Disponível em: <http:// anabad.org/noticias-anabad/28-bibliotecas/6957diez-anos-de-europeana>. Acesso em: 8 dez. 2018.

AGENJO, X.; CAMPILLEJO, M. El observatorio de la digitalización en Europa. Desafíos y oportunidades de las Ciencias de la Información y la Documentación en la era digital. Anais... In: VII ENCUENTRO IBÉRICO EDICIC 2015 (MADRID, 16 Y 17 DE NOVIEMBRE DE 2015). Madrid: Universidad Complutense de Madrid, 17 nov. 2015 Disponível em: <http://edicic2015.org.es/>. Acesso em: 8 dez. 2018

COMISIÓN EUROPEA. Recomendación de la Comisión de 27 de octubre de 2011 sobre la digitalización y accesibilidad en línea del material cultural y la conservación digital. (2011/711/UE), out. 2011. Disponível em: <https://eur-lex.europa. eu/legal-content/ES/TXT/HTML/?uri=CELEX:320 $11 \mathrm{H} 0711$ \&qid $=1544125153372 \&$ from $=E S>$. Acesso em: 6 dez. 2018

COMISIÓN EUROPEA. Getting cultural heritage to work for Europe: Report of the Horizon 2020 expert group on cultural heritage. [s.l.] Dirección General para la Investigación y la Innovación, abr. 2015. Disponível em: <https://publications.europa.eu/ en/publication-detail/-/publication/b01a0d0a-2a4f4de0-88f7-85bf2dc6e004/language-en/formatPDF/source-31403758>. Acesso em: 7 dez. 2018.

COMISIÓN EUROPEA. Implementation of Commission Recommendation on the digitisation and online accessibility of cultural material and digital preservation: Progress report 2013-2015. [s.l: s.n.]. Disponível em: <http://ec.europa. eu/information_society/newsroom/image/document/2016-43/2013-2015_progress_report_18528.pdf>. Acesso em: 8 dez. 2018.

COMISIÓN EUROPEA. Summary report of the public consultation on Europeana, Europe's Digital Platform for Cultural Heritage. Disponível em: <https://ec.europa.eu/digital-single-market/en/ news/summary-report-public-consultation-europeana-europes-digital-platform-cultural-heritage>. Acesso em: 20 dez. 2018a.

COMISIÓN EUROPEA. Comunicación de la Comisión al Parlamento Europeo, al Consejo, al Comité Económico y Social Europeo y al Comité de las Regiones. Una Nueva Agenda europea para la cultura. $\{S W D(2018) 167$ final\}, maio 2018b. Dispo- nível em: <https://eur-lex.europa.eu/legal-content/ ES/TXT/HTML/?uri=CELEX:52018DC0267\&from= EN>. Acesso em: 6 dez. 2018

CONSEJO EUROPEO. Conclusiones del Consejo, de 20 de noviembre de 2008, relativas a la biblioteca digital europea EUROPEANA. (2008/C 319/07), 20 nov. 2008. Disponível em: <https:// eur-lex.europa.eu/legal-content/ES/TXT/HTML/? uri=CELEX:52008XG1213(04)\&qid=1544637825 760\&from $=E S>$. Acesso em: 12 dez. 2018

CONSEJO EUROPEO. Directiva 2012/28/UE del Parlamento Europeo y del Consejo de 25 de octubre de 2012 sobre ciertos usos autorizados de las obras huérfanas, 2012. Disponível em: <https://eur-lex.europa.eu/legal-content/ ES/TXT/HTML/?uri=CELEX:32012L0028\&from= ES>. Acesso em: 7 dez. 2018

CONSEJO EUROPEO. Conclusiones del Consejo, de 21 de mayo de 2014, sobre el patrimonio cultural como recurso estratégico para una Europa sostenible. 2014/C 183/08, 21 maio 2014. Disponível em: <https://eur-lex.europa.eu/legalcontent/ES/TXT/HTML/?uri=CELEX:52014XG06 14(08)\&qid=1544641890754\&from=ES>. Acesso em: 12 dez. 2018

CONSEJO EUROPEO. Conclusiones del Consejo sobre la función de Europeana en el acceso, la visibilidad y el uso digitales del patrimonio cultural europeo. (2016/C 212/06), 14 jun. 2016. Disponível em: <https://eur-lex.europa.eu/legalcontent/ES/TXT/HTML/?uri=CELEX:52016XG06 14(02)\&qid=1544638021969\&from=ES>. Acesso em: 12 dez. 2018

ENUMERATE. Survey Report on Digitisation in European Cultural Heritage Institutions 20144. Disponível em: <https://www.egmus.eu/fileadmin/ ENUMERATE/documents/ENUMERATE-Digitisation-Survey-2014.pdf>. Acesso em: 8 dez. 2018.

EUROPEANA. The Europeana Licensing Framework. [s.l: s.n.]. Disponível em: <https://pro. europeana.eu/files/Europeana_Professional/Publications/Europeana\%20Licensing\%20Framework. pdf>. Acesso em: 20 dez. 2018.

EUROPEANA. Europeana Strategy 2020: 'We transform the world with culture'. Disponível em: $<$ http://strategy2020.europeana.eu/>. Acesso em: 20 dez. 2018.

EUROPEANA. Marco de publicación de Europeana: cuanto más das más recibes. [s.l: s.n.]. Dis- 
ponível em: <http://travesia.mcu.es/portalnb/jspui/ handle/10421/8991>. Acesso em: 20 dez. 2018.

EUROPEANA. Guía de publicación de Europeana v1.6. [s.l: s.n.]. Disponível em: <http://travesia.mcu.es/portalnb/jspui/handle/10421/8995>. Acesso em: 20 dez. 2018a.

EUROPEANA. Modelo de datos de Europeana (EDM). Directrices de Asignación v 2.4. [s.I: s.n.]. Disponível em: <http://travesia.mcu.es/ portalnb/jspui/handle/10421/8999>. Acesso em: 20 dez. 2018 b.

EUROPEANA. Estrategia de Contenidos de Europeana: contenidos adecuados para el usuario, en el momento justo. [s.l: s.n.]. Disponível em: <http://travesia.mcu.es/portal$\mathrm{nb} / \mathrm{jspui} /$ handle/10421/9006>. Acesso em: 20 dez. 2018.

GONZÁLEZ, S. Aportación de Hispana a Europeana HISPANAPRO, 13 ago. 2018. Disponível em: $<$ http://hispanapro.mecd.es/aportacion-de-hispana-a-europeana-2/>. Acesso em: 20 dez. 2018

JAN NAUTA, G.; HEUVEL, W. VAN DEN; TEUNISSE, S. Report on ENUMERATE Core Survey 4. [s.I: s.n.]. Disponível em: <https://pro.europeana.eu/files/Europeana_Professional/Projects/Project_list/ENUMERATE/deliverables/DSI-2_Deliverable\%20D4.4 Europeana Report\%20on\%20 ENUMERATE $\% \overline{20}$ Core $\% 20$ Survey $\% 204$.pdf>. Acesso em: 18 dez. 2018.

MAK, M.; BRINKMAN, C. Europeana Channels: Summary report Concept test. [s.I: s.n.]. Disponível em: <https://pro.europeana.eu/files/Europeana Professional/Projects/Project_list/Europeana_Version3/Documents/20141016-summary-report-europeana-channels-concepttest-v1.pdf>.

MINISTERIO DE CULTURA. Política de Gestión de Documentos Electrónicos del Ministerio de Educación, Cultura y Deporte - Ministerio de Educación, Cultura y Deporte. Disponível em: <http:// www.mecd.gob.es/cultura-mecd/areas-cultura/archivos/recursos-profesionales/documentos-electronicos.html>. Acesso em: 26 fev. 2017.

PARLAMENTO EUROPEO. INFORME sobre «Europeana - Ios próximos pasos» - A70028/2010. [s.l: s.n.]. Disponível em: <http:// www.europarl.europa.eu/sides/getDoc. do?pubRef=-//EP//TEXT+REPORT+A 7 2010-0028+0+DOC+XML+V0//ES>. Acesso em: 12 dez. 2018.
PARLAMENTO EUROPEO; CONSEJO EUROPEO. Directiva 2003/98/CE del Parlamento Europeo y del Consejo, de 17 de noviembre de 2003, relativa a la reutilización de la información del sector público., 17 nov. 2003. Disponível em: $<$ https://eur-lex.europa.eu/legal-content/ES/TXT/ HTML/?uri=CELEX:32003L0098\&qid=154529621 8127\&from=ES>. Acesso em: 20 dez. 2018

PARLAMENTO EUROPEO; CONSEJO EUROPEO. Propuesta de Directiva del Parlamento Europeo y del Consejo relativa a la reutilización de la información del sector público (versión refundida). COM(2018) 234 final, abr. 2018c. Disponível em: <https://eur-lex.europa.eu/legal-content/ES/TXT/ HTML/? uri=CELEX:52018PC0234\&from=EN>. Acesso em: 7 dez. 2018

RODRÍGUEZ-MATEOS, D.; HERNÁNDEZ-PÉREZ, T. Televisión social en series de ficción y nuevos roles del documentalista audiovisual: el caso de 'El Ministerio del Tiempo'/Social TV on fiction series and new roles for a media librarian:'El Ministerio del Tiempo'as a case study. index. comunicación, v. 5, n. 3, p. 95-120, 2015.

UNESCO. Tangible Cultural Heritage. Disponível em: <http://www.unesco.org/new/en/cairo/culture/tangible-cultural-heritage/>. Acesso em: 12 dez. 2018.

\section{Recebido em 20/12/2018 Aprovado em 24/01/2019}

I María-Antonia García-Moreno. Dpto. de Biblioteconomía y Documentación, Universidad Complutense de Madrid, Espanha. Contato: magm@ucm.es

II Tony Hernández-Pérez. Dpto. de Biblioteconomía y Documentación, Universidad Carlos III de Madrid, Espanha. Contato:. tony@bib.uc3m.es 